\title{
Psychologie et implantologie
}

\section{Psychology and implantology}

«On peut convaincre les autres par ses propres raisons, on ne les persuade que par les leurs. »

Joseph Joubert, Les Pensées, 1838

\section{MOTS-CLEFS :}

- Accueil, réception équations psychologiques trois consultations équipe thérapeutique

\section{KEYWORDS:}

- Welcome, reception, psychological equations, 3 visits,

therapeutic team

\section{Résumé}

Confrontés à une société de plus en plus consumériste et face aux défis thérapeutiques, économiques et médiatiques liés ò nos exercices, notre crédibilité doit passer par plus de savoir-faire.

La dictature de l'image et de la communication dans un contexte de performance et de concurrence va impliquer une autre dimension : le faire-savoir, et ce, dans le respect de notre déontologie.

La pratique intuitive de nouveaux instruments de communication, les concepts d'expression orale et non verbale, l'abord maîtrisé du visuel et de la gestuelle vont aller dans le même sens : poser et mieux proposer l'alternative implantaire, eu égard aux demandes des patients.

\section{Abstract}

In an increasingly consumerist society and faced with the therapeutic and economic challenges and media exposure associated with our profession, our credibility has to be based on more than just know-how.

The reigning culture of image and communication with its emphasis on performance and competition brings another dimension into play: how-to-know, and this must be accomplished without compromising our professional ethics.

The intuitive implementation of new communication tools and concepts of oral and nonverbal expression and a proper understanding of the visual and the gestural all work towards the same goal: to more effectively explain and propose the implant option, in consideration of the demands of our patients.
AOS 2014;268:36-42

DOI: $10.1051 / \mathrm{aOs} / 2014208$

(C) EDP Sciences 2014

Jean-Pierre CHETRY, Membre associé national de l'Académie nationale de
chirurgie dentaire




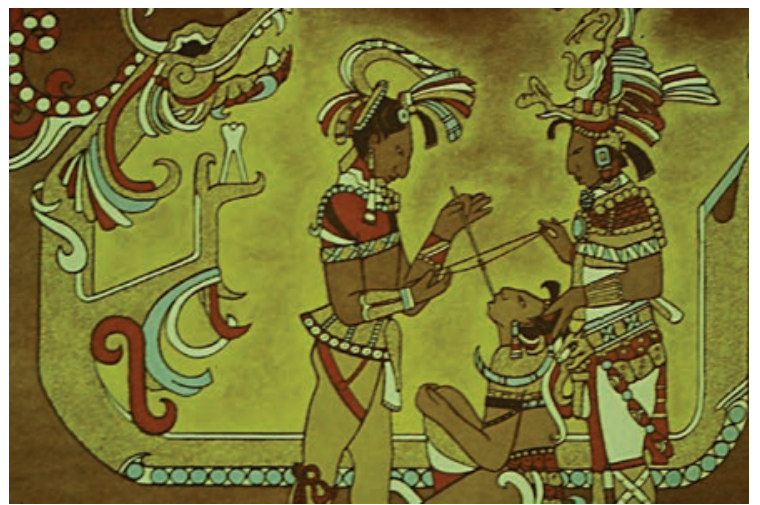

$\Delta$ Fig. 1.

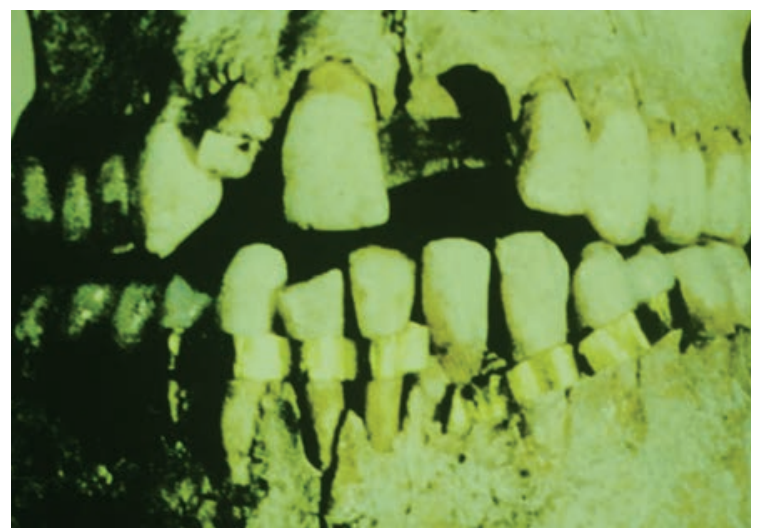

$\Delta$ Fig. 2.

\section{INTRODUCTION}

Depuis la nuit des temps, le désir de paraître, plaire et séduire a été une préoccupation constante : choix des parures, des vêtements, des coiffures mais aussi respect d'une certaine intégrité physique et, pour ce qui nous concerne, celle de la cavité orale (fig. 1).

Carrefour de la communication et du langage, elle véhicule notre intégration à la vie sociale.

Confrontée au regard d'autrui, elle vise à s'identifier aux normes et modèles en vigueur, et tout se qui s'en éloigne peut être considéré comme source de dépréciation personnelle.

Les Étrusques, qui 2500 ans avant J.-C. tenaient une vie sociale très riche et structurée, anticipaient la perte des dents par des bandelettes d'or reliant des dents mobiles.

L'examen anatomique (fig. 2) recensait essentiellement des crânes féminins, connotant un souci de séduction et d'esthétique.

Chez l'homme,l'approche psychologique est différente. La dent allie force, agressivité et virilité : « avoir la dent dure ", « montrer les dents ». A contrario, la chute des dents peut signifier une véritable mutilation par perte de la force physique et de la diminution de la fonction. Intervenant au niveau de la sphère centrale, siège de notre sensibilité et de nos émotions, nous allons être confrontés par prolongement au problème de l'expression douloureuse.

Esthétique, fonction, douleur, problèmes de santé, de statut social, pression de l'entourage, c'est dire l'importance du premier contact au cours duquel il faudra cerner les besoins prioritaires et précis du patient. Ayant la chance exceptionnelle d'être spontanément sollicités par nos patients dans nos cabinets, nous devons privilégier plusieurs dimensions qualitatives.

\section{Qualité de l'accueil téléphonique}

Premier maillon dans la longue chaîne de la communication, il constitue un média-témoin redoutable, reflétant d'emblée l'image du cabinet.

Sourire au téléphone, diplomatie de l'écoute, maîtrise des réponses, c'est le rôle d'une réceptionniste aguerrie face à un florilège trop souvent rabattu au bout du fil.

I Ne quittez pas!

Merci de rester en ligne.

Je ne sais pas si le Dr.

Je vérifie et vous confirme...

1 C'est pourquoi ?

Quel est l'objet de votre appel ?

I Expliquez-moi de quoi il s'agit...

En quoi puis-je vous aider?

IJe vais voir si je peux le déranger.

Je vérifie s'il peut vous prendre.

1 C'est de la part de qui ?

Qui dois-je annoncer?

I Faire la commission.

Transmettre le message.

\section{Qualité de la réception}

Initiée par la secrétaire, elle allie disposition d'accueil et empathie. La gestion partagée du dossier administratif engendre un colloque singulier qui, nourri des réflexions ou des préoccupations du patient, va déjà inciter la secrétaire à des réponses rassurantes. De par ce passage obligé, nous aurons en amont une idée de son profil psychologique et, dès lors, nous pourrons mieux adapter en retour notre communication avant de recevoir notre patient.

\section{Qualité du cadre d'exercice}

Se référant au visuel retenu à $87 \%$ par rapport au verbal, le lieu professionnel doit imprimer l'image de structures claires, aérées, vitrées laissant deviner chaîne de stérilisation, salle d'intervention, etc.

L'arrêt de la Cour de cassation du 28 février 2002 stipule pour l'implantologie une installation convenable et des moyens techniques suffisants (fig. 3). 


\section{Qualité de la première impression}

Il s'agit d'un instant de vérité basé sur la règle des 3 vingt :

$120 \mathrm{~cm}^{2}$ du visage;

120 premiers mots ;

120 premières secondes.

Premier message à délivrer : le sourire. Il y a lieu de le véhiculer en disposant du patient dans le salon d'attente et par un serrement de main, déjà initier le premier ancrage physique.

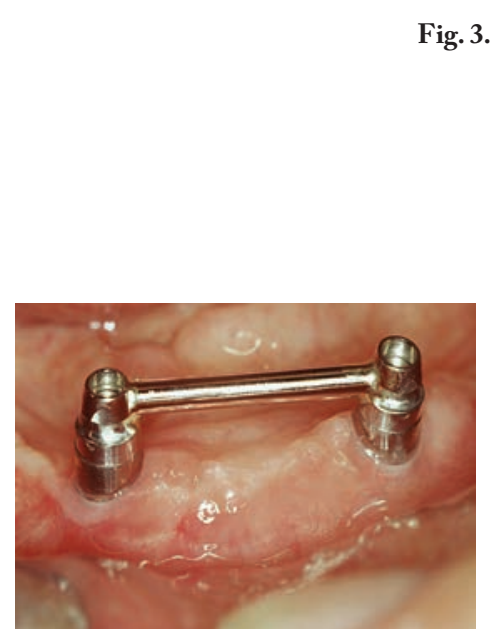

A Fig. 4.

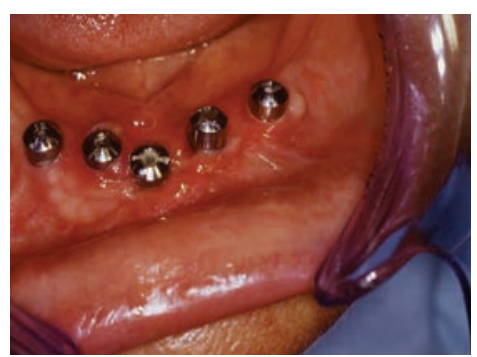

$\Delta$ Fig. 5.

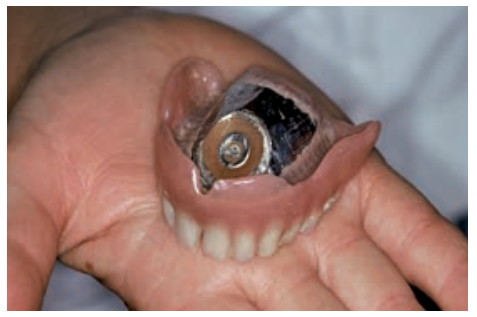

$\Delta$ Fig. 7.

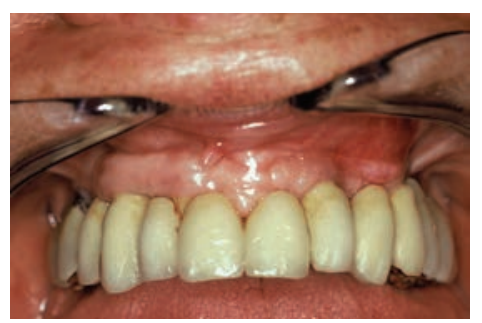

$\Delta$ Fig. 9.
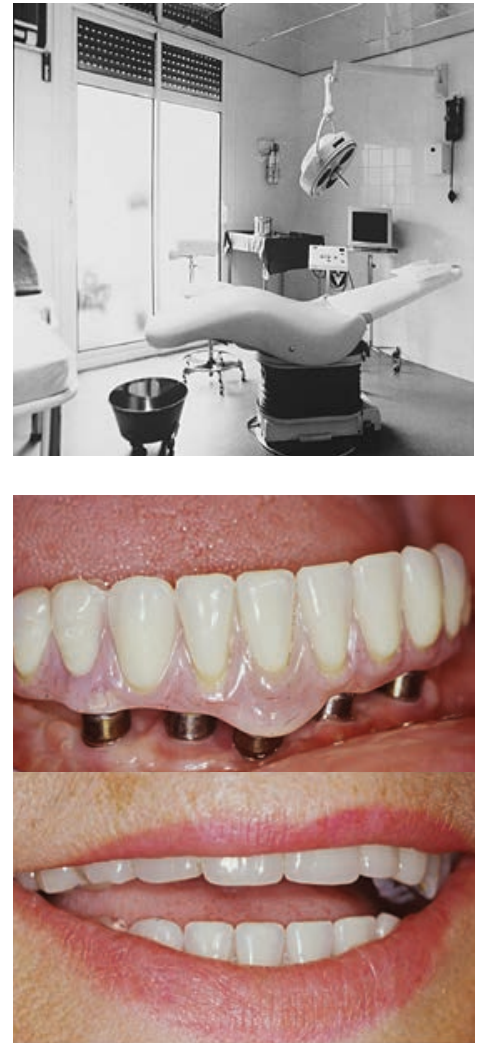

$\Delta$ Fig. 6.

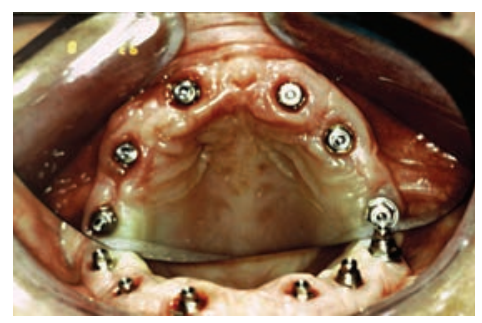

A Fig. 8.

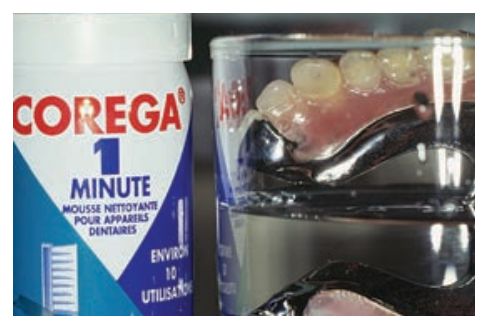

$\triangle$ Fig. 10.
L'entretien devra éviter la barrière du bureau et préférer un espace côte à côte pour assurer une distance de confort, rapprochant la communication.

À l'écoute, nous allons à la découverte des attentes, besoins et motivations.

L'attente fait appel à l'affectif, le besoin à une demande matérielle et concrète de dents.

La motivation, plus liée au psychologique et au subjectif, vise plutôt «à être bien dans sa bouche ».

L'addition des attentes, des besoins et des motivations réalise aussi l'équation psychologique du patient.

\section{Équations psychologiques}

I Chez la personne âgée, avec une impotence masticatoire mandibulaire, 2 implants avec barre ou boutons pression assureront, à moindre coût, rétention, stabilisation et sustentation de la prothèse, voire même de l'ancienne modifiée (fig. 4).

I Il peut aussi s'agir d'effacer les effets de l'âge par effondrement des étages faciaux lié à une prothèse totale instable : sillons nasogéniens creusés, lèvres pincées, profil labio-mentonnier rétréci (fig. 5).

Une prothèse ostéoancrée fixée sur 5 implants changera la vie de la patiente sur le plan fonctionnel par un potentiel masticatoire multiplié par quatre. La transformation esthétique sera de mise avec les lèvres plus ourlées, une remise en norme et en tension de toute la musculature du visage réalisant un véritable lifting anatomique. Si l'on ajoute la sécurité retrouvée, le bénéfice psychologique reste sans égal (fig. 6).

I Pour ce cas (fig. 7), il y a volonté de la patiente d'éradiquer colle et ventouse, responsables d'un traitement anxiolytique en plus d'une addiction nicotinique.

Une réalisation fixe implantaire maxillaire visant à supprimer ces artifices constitue pour elle une alternative fiable et durable.

Confiance et confort, tout concourt à annuler son traitement psychotrope (fig. 8 et 9 ).

1 Ce peut être le souci de se séparer d'une prothèse mobile. Le fait de la retirer ne serait-ce que pour la nettoyer (fig. 10) nous rappelle qu'une partie de notre intimité flotte, immerge et se reflète dans le verre d'eau.

I Pour les femmes, le ressenti reste une blessure quotidienne.

Trois implants et une réalisation fixe constituent la meilleure réponse face à un handicap mal vécu (fig. 11 et 12).

I Chezles jeunes, la nouveauté, la rigueur du système les invitent naturellement à cette thérapeutique.

Si l'extraction signifie de façon péjorative l'échec du traitement conservateur (fig. 13), l'implant, en tant qu'acte de reconstruction, s'associe chez ces patients à un gage de modernité en phase avec leur âge (fig. 14 et 15). 


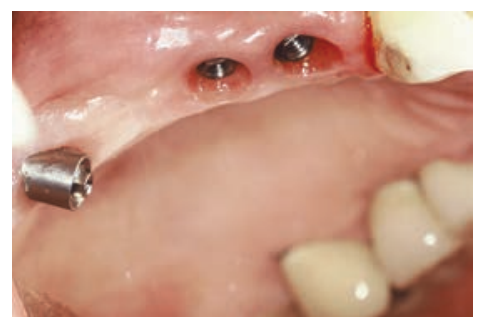

$\Delta$ Fig. 11.

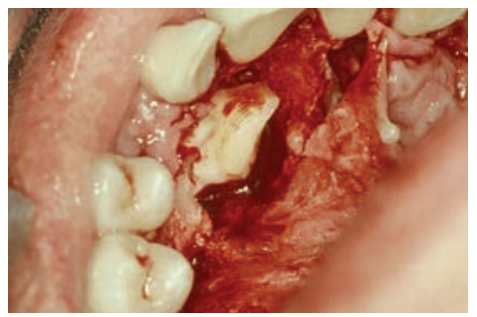

$\triangle$ Fig. 13.

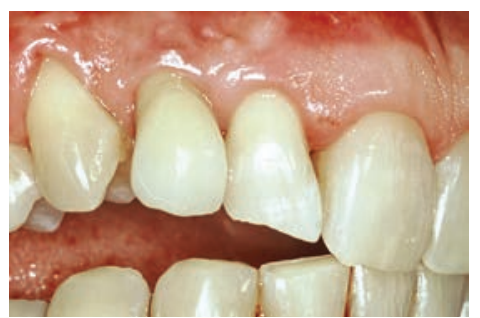

$\triangle$ Fig. 15.

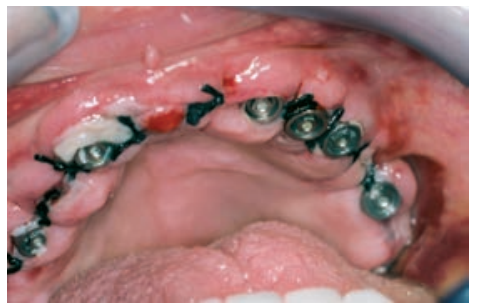

$\Delta$ Fig. 17.

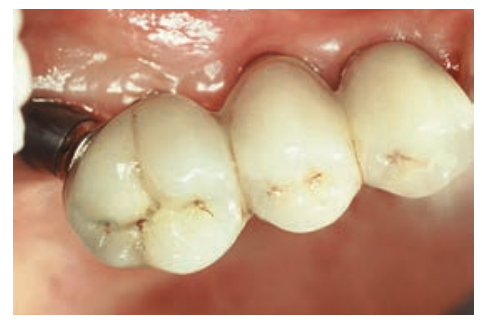

$\Delta$ Fig. 12.

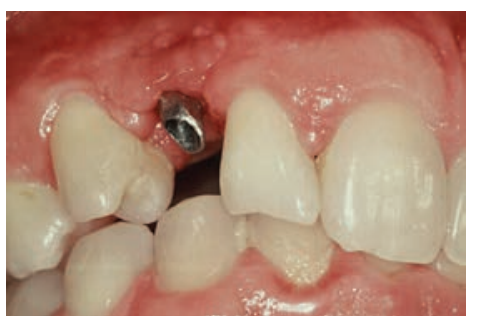

$\triangle$ Fig. 14.

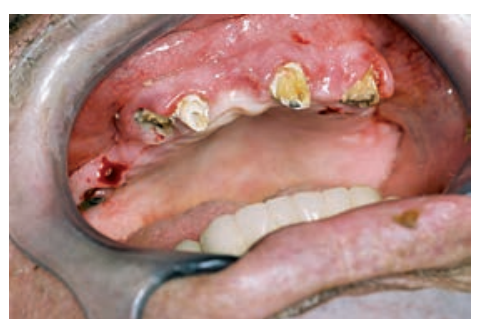

$\Delta$ Fig. 16

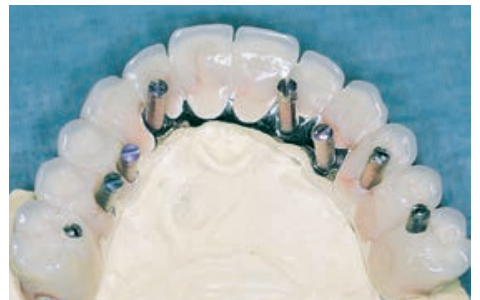

$\triangle$ Fig. 18.

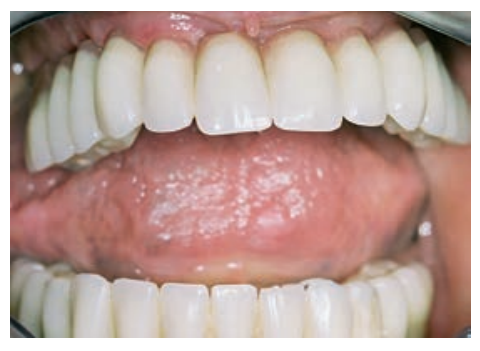

Fig. 19.

I Parfois, le temps de cicatrisation post-extractionnel, l'attente de l'ostéointégration implantaire et l'inconfort des prothèses transitoires peuvent dissuader le patient.

Lextraction-implantation, couplée à une mise en charge immédiate, en faisant renaitre de nouvelles dents à 24 heures, reste un argument fort dans l'arbre décisionnel (fig. 16 à 19).

Depuis le 25 février 1997, notre devoir d'information doit porter certes sur la solution implantaire, mais aussi sur les techniques dites alternatives. Outre le consentement éclairé, le nombre de rendez-vous, leur espacement et la durée sont vecteurs de l'information par présomption et déduction sur le plan médico-légal (arrêt de la cour d'appel de Poitiers du 12 janvier 2011).

Dès lors, trois consultations doivent s'atteler à cette jurisprudence.

\section{PREMIĖRE CONSULTATION}

Il y a lieu d'éviter tout bruit parasite (téléphone, etc.) et tout dérangement pouvant interférer dans l'entretien.

Faire en sorte de bannir les questions fermées, brutales et sèches qui appellent une réponse par oui ou par non, sans faire avancer le débat.

Préférer les questions ouvertes, informatives et interrogatives où le patient va s'ouvrir avec son vocabulaire et ses valeurs. Dès lors, l'espace de communication étant élargi, il pourra mieux expliquer ses attentes, refuser de renouveler des expériences antérieures, et par là-même, être amené à les critiquer de lui-même.

Ne pas hésiter à reformuler et à clarifier pour être en phase avec ses propos : «Si je comprends bien, si je résume bien, vous soubaitez... ", montrant que le message a été bien reçu.

Instiller parfois des doses d'approbation sur le vécu: "Oui bien sûr, je me mets à votre place... ".

Grâce à ces échanges, le praticien commence à « rentrer» dans la personnalité et le quotidien du patient. C'est le début de l'empathie, élan d'identification vers la personne qui vise à susciter confiance et future adhésion.

L'examen clinique intra-oral pouvant être ressenti comme une intrusion précoce dans son intimité, il y a plutôt lieu de commencer par une palpation douce des téguments externes, des ATM, contrôle de l'ouverture, de la fermeture de la bouche, déviation, etc.

Au constat dentaire, ne pas hésiter à faire revenir le patient sur son état pour mieux évaluer sa capacité ancienne de coopération et le degré actuel de motivation. Quoi qu'il en soit, ne jamais critiquer ou culpabiliser, préférer rassurer et rappeler que nous savons faire même dans les situations délicates.

À ce stade, procéder à une prise d'empreintes pour des modèles d'étude, prescrire un éventuel scanner en ayant déjà pris en compte d'éventuelles contre-indications générales ou locales.

Même quand le plan de traitement est évident, temporiser et remettre la décision thérapeutique, car, après réflexion, elle peut changer.

Ce délai suscite généralement désir et espoir chez le patient. 


\section{DEUXIÈME CONSULTATION}

Éviter de découvrir le scanner devant le patient. Trois secondes d'examen découlent de la réflexion, au-delà doute et inquiétude risquent de s'installer. Préférer une anticipation de cette étude pour mieux l'expliciter à ce stade.

\section{Outils pédagogiques}

"Voir c'est croire .»

La caméra intrabuccale en tant qu'outil de codiagnostic, tout en projetant l'image et la maitrise technique du cabinet, va "sublimer » l'etat buccodentaire en grandissant (fig. 20) le motif de la consultation.

Son usage aux États-Unis a permis de multiplier par deux le taux d'acceptation des propositions. Lenregistrement des images en figeant l'état antérieur pourra servir d'argument médico-légal et de référentiel comparatif pour le patient et son entourage à la fin du traitement.

\section{Modèles d'étude (fig. 21)}

Montés sur articulateur et tenus dans les mains du patient, ils matérialisent concrètement létat de ses édentations. Un montage esthético-fonctionnel préfigurant les futures dents atteste que le projet avance concrètement.

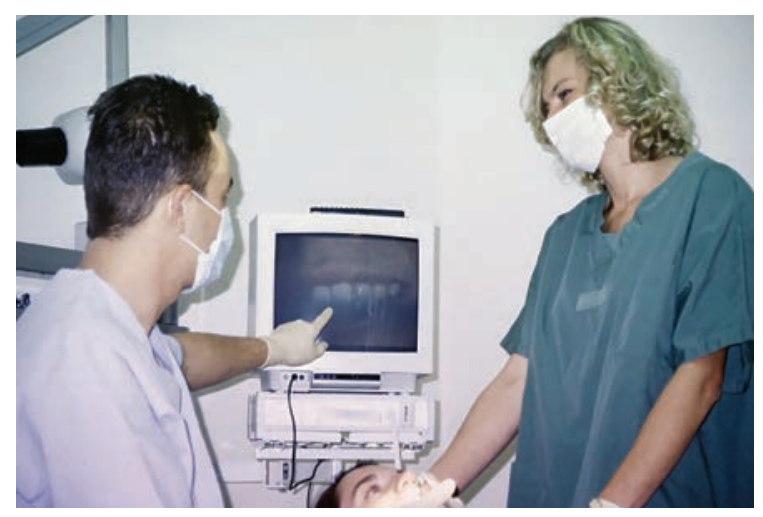

$\triangle$ Fig. 20.

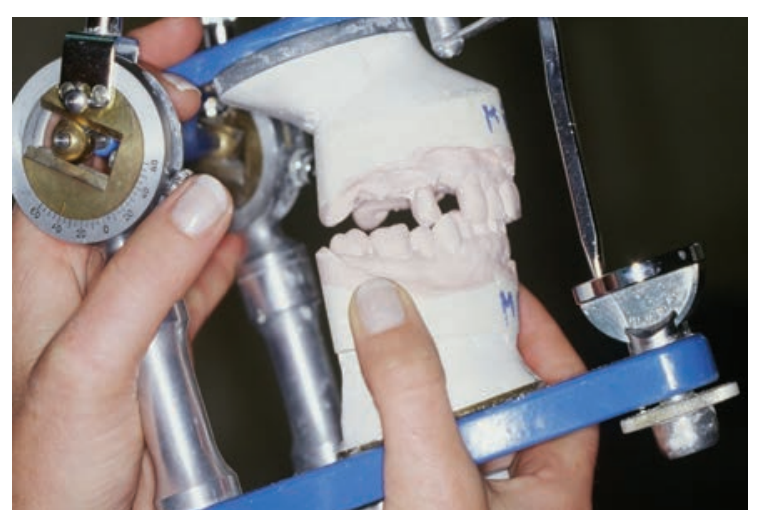

A Fig. 21.
Face aux techniques d'imagerie médicale moderne et aux supports audiovisuels traditionnels, un simple dessin fait devant lui et pour lui peut conforter notre argumentation en la personnalisant.

Celle-ci ne peut se faire au futur qui n'est jamais certain, ni au conditionnel teinté d'aléas et d'hésitations. Préférer l'utilisation du présent, temps de l'action et de l'efficacité.

Il faut faire comprendre au patient « qu'il a déjà les implants, qu'il les utilise et qu'il en est satisfait ».

\section{Proposition}

Axée sur un véritable travail d'étude en amont bien expliqué au patient, une sémantique adaptée s'impose. "À l'examen clinique de votre bouche, à l'étude des modèles en plâtre, vu les résultats du scanner et compte tenu de votre motivation, nous pouvons envisager ensemble..." Le «nous" a une capacité d'entraînement dynamique vis-à-vis du « je » professionnel et souvent trop magistral.

Le «pouvons envisager» : rien n'est imposé.

«ensemble » : c'est toute une équipe qui participe au traitement impliquant nécessairement la collaboration du patient.

\section{Mots clés}

I Réalisation FIXE qui va fonctionner comme des dents naturelles.

Dans notre inconscient, tout ce qui est fixe soigne de l'intérieur et signe une approche thérapeutique définitive.

$A$ contrario, tout ce qui est mobile intervient de l'extérieur avec une connotation de provisoire et de transitoire.

I La satisfaction d'un retour à une situation antérieure à la pathologie présente.

I Les avantages dans la sécurité, le confort et la confiance retrouvés.

I Les preuves : fixité assurée par scellement doux ou vissage.

I La garantie : possibilité de démontage ou de dévissage en cas de réactualisation, de réparation ou de remplacement.

Dans certains cas, où le message thérapeutique n'est pas bien compris, ne pas hésiter à convoquer un patient bénéficiaire d'un traitement jumeau.

Devant ce nouvel invité, procéder à des questions interrogatives pour accentuer l'effet levier :

1 Question ouverte de relance : Quien pensez-vous?" I Question ouverte de stimulation et de simulation: « $S i$ nous obtenons ce résultat, quelle serait votre réaction?" I Reformulation et clarification: "Si nous résumons, c'est ce que vous attendez?"

I Technique des OUI préalables: «Nous sommes donc d'accord sur le projet?" 
Ces interpellations suscitent généralement un déclenchement d'émotions qui invitent à « la possession par anticipation ".

Dans nos réponses, il y a lieu dêtre préparés aux traditionnelles questions concernant les contre-indications éventuelles, l'âge, la douleur, la durée, le rejet, le calendrier thérapeutique, l'intérêt et les inconvénients des techniques alternatives.

La réponse au coût vient en dernier. Faire comprendre qu'il s'agit d'un dispositif médical de santé unique, intellectuellement réfléchi et conçu spécialement pour lui, en un seul exemplaire et non transposable à autrui. Loin dêtre une dépense à fonds perdus, il s'agit d'un investissement qui va enrichir sa propre personnalité et le rendre pleinement propriétaire de sa bouche.

La persuasion et les réponses aux objections s'inscrivent dans plusieurs attitudes :

1 l'addition : additionner tous les avantages dus à la technique implantaire;

I la soustraction : en retrancher tous les bénéfices;

I la division : ramener les honoraires à une dépense journalière pendant une durée déterminée (tabac, etc.).

I Éviter toute précipitation dans nos réponses. Un temps d'arrêt signe toujours une réflexion plus personnelle et personnalisée.

\Éviter les réponses dites «barbelés " qui ont tendance à « griffer »l'interlocuteur. C'est pourquoi :

Ine pas fuir une objection en coupant la parole mais laisser le patient s'exprimer car une objection peut en cacher une autre sur laquelle nous pouvons rebondir;

I ne pas rejeter une remarque car toute remarque est bienvenue même si elle ne s'applique pas au cas;

Ine pas insister dans nos réponses car une explication longue signe un problème important que le patient a bien fait d'évoquer.

\section{TROISIĖME CONSULTATION}

Elle vise, en même temps qu'une réponse à des demandes légitimes de clarification qui nous sont soumises, à être préparés à la décision du patient.

\section{Refus du devis}

Manque de motivation, perte de confiance, préjugés liés aux expériences antérieures, problèmes personnels ou professionnels : ne jamais marquer notre déception. Éviter les « faux appels à la peur » en cas de renoncement mais informer le patient de l'évolution prévisible et des conséquences en cas de non-traitement dans un langage qui lui est accessible.

\section{Accord sur le devis}

Éviter les «faux appels à la confiance » par des superlatifs concernant la technique, les futurs résultats, voire même nos compétences professionnelles.

Faire signer en silence le document en double exemplaire en notant :

Ile temps de validité;

$\checkmark$ les éventuelles prises en charge par la Sécurité sociale et les mutuelles;

I conserver les devis concernant les techniques alternatives qui signent une information pertinente.

\section{ACTEURS DANS L'ÉQUIPE THÉRAPEUTIQUE (fig. 22)}

\section{Patient}

C'est le centre d'intérêt permanent. Ne pouvant faire son bonheur malgré lui, il y lieu d'avoir testé son état de motivation, de coopération et d'engagement financier.

\section{Assistante}

En dehors de son aide opératoire, elle constitue le ciment et l'interface entre tous les acteurs.

\section{Chirurgien}

Il se doit d'éviter les mots qui «écorchent » : piqûre, arracher la dent, cureter la gencive, etc. et préférer des formules plus douces : insensibiliser la zone, supprimer le foyer infectieux, remodeler les tissus mous, etc. Pendant l'intervention sous champ opératoire, parler, réconforter et rassurer le patient sur le bon déroulement.

Artisans des fondations implantaires, nous évoluons dans la zone rouge noyée de sang, de stress et d'appréhension, peu propice à gratifier notre acte chirurgical. L'attente du patient réside essentiellement dans la partie noble et émergente de l'implantologie : les dents.

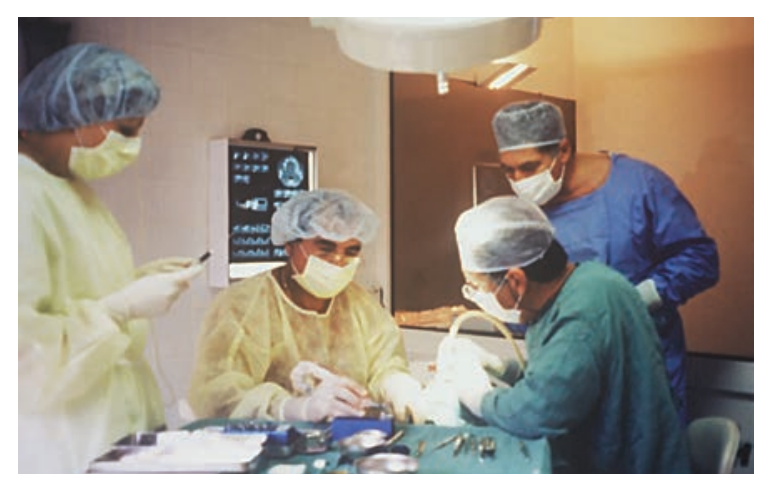

$\triangle$ Fig. 22. 


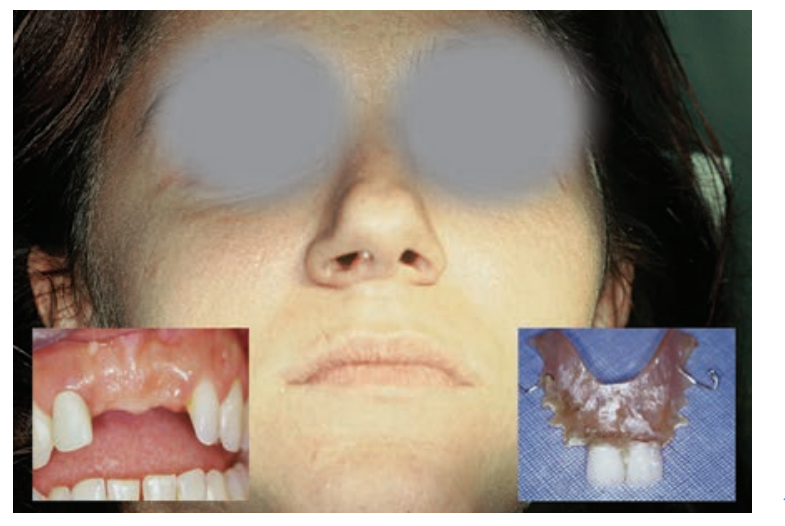

4 Fig. 23.

$\nabla$ Fig. 24.

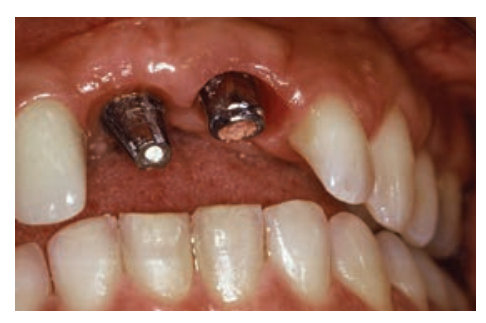

$\nabla$ Fig. 26.

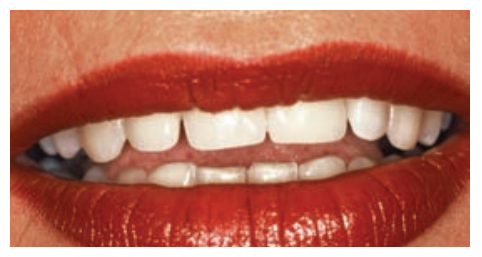

V Fig. 25.

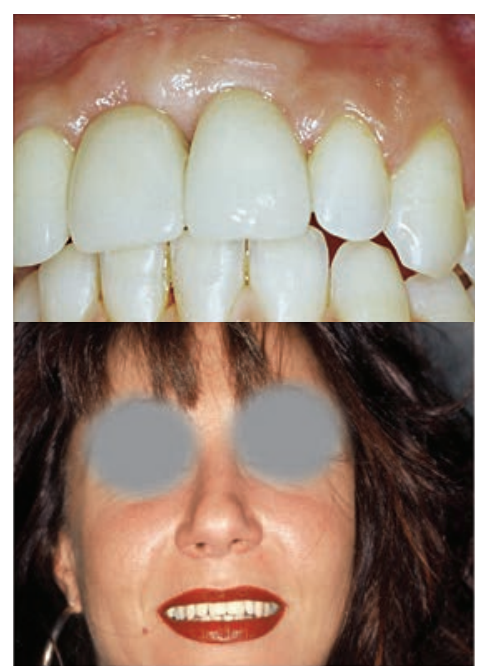

Ce peut être par délégation de la responsabilité d'un autre praticien, le prothodonctic.

\section{Prothodontic}

Le prothodontic est uniquement en charge de la partie prothétique.

Dès lors que la finalité fonctionnelle et esthétique lui incombe, en cas de succès, la plus-value psychologique ira de pair avec ses compétences.

\section{Technicien de laboratoire}

Dans certains cas complexes, la présence et les observations du technicien peuvent irriguer favorablement notre plan de traitement et optimiser la liaison laboratoire-cabinet lors des essayages et des prises de teinte. Entouré, conseillé, rassuré par une équipe de spécialistes entièrement dévolus à son service, le patient au centre du traitement a vocation à " présider » le déroulement des séances.

La vertu de ces protocoles, outre la finalité thérapeutique, présente un intérêt majeur : crédibiliser et justifier nos honoraires.

Hormis cette communication partagée dans l'équipe thérapeutique, nous pouvons secrètement être confrontés à des considérations d'ordre intimiste surtout chez la femme. En effet, en plus du langage, la cavité orale décline d'autres fonctions : le manger, le boire, le plaisir, l'amour, la sexualité. Dans certains cas, la maladie parodontale ou l'accident traumatique peuvent l'entraver.

\section{Patiente, 22 ans}

IExpulsion des 2 incisives centrales maxillaires, secondaire à une chute en rollers.

I Tristesse dans le visage, lèvres fermées occultant une prothèse amovible en résine dégradée, odorante et réparée à plusieurs reprises (fig. 23).

I Pose de 2 implants et de 2 piliers (fig. 24).

$\checkmark$ Mise en place de 2 coiffes en zircone (fig. 25) avec en retour une expression orale libérée par le sourire, lèvres ourlées et maquillées (fig. 26) et, en confidence, la pratique retrouvée du french kiss!

Quand on sait que dans ce cas, il y a : la mobilisation de 22 muscles ; des échanges d'eau, de sels, de bactéries et d'enzymes ; la libération d'endorphines et de testostérone ; un éveil des sens (pics d'adrénaline, fluidification des muqueuses, pouls augmenté, perte de calories, mélange de goût, ouie, respiration et succion, odorat et libération de phéromones), c'est une véritable révolution physiologique qui prend corps. Si l'on ajoute l'impact visuel vis-à-vis du regard d'autrui, l'acte implantaire constitue dans ce cas le traitement le plus bénéfique sur le plan psychologique.

\section{CONCLUSION}

L'art implantaire, thérapeutique plurielle au carrefour de nombreuses disciplines, chirurgie, parodontologie, prothèse, esthétique, psychologie, implique une autre dimension non retenue dans nos formations : la communication.

Tout au long de notre parcours professionnel de plus en plus exigeant, il y a lieu de tester nos capacités d'accueil, d'écoute et d'empathie.

À ce prix, nous pourrions mieux initier des forces d'information, d'argumentation et de proposition. Tenus en plus à une certaine « obligation de résultat » souvent subjective, tant sur le plan fonctionnel qu'esthétique, il est certain qu'un environnement psychologique adapté s'impose.

A ce titre et dans ces conditions, nous pourrions sagement optimiser nos exercices et éviter des contresens générateurs de futurs conflits.

$P S$ : cet article est tiré d'un cours dispensé dans de nombreux DU et formations post-universitaires. 